\title{
ON THE PROPAGATION OF CALCIUM WAVES IN AN INHOMOGENEOUS MEDIUM*
}

\author{
JAMES SNEYD ${ }^{\dagger}$ AND JONATHAN SHERRATT ${ }^{\ddagger}$
}

\begin{abstract}
Although the exact details are disputed, it is well established that propagating waves of increased intracellular free $\mathrm{Ca}^{2+}$ concentration arise from a positive feedback or autocatalytic mechanism whereby $\mathrm{Ca}^{2+}$ stimulates its own release. Most previous modeling of the propagation of $\mathrm{Ca}^{2+}$ waves has assumed that the sites of autocatalytic $\mathrm{Ca}^{2+}$ release, the activation sites, are homogeneously distributed through the cytoplasm. We investigate how the spacing and size of the activation sites affect the existence and speed of propagating calcium waves. We first study the simplest model of an excitable system to obtain analytic estimates of the critical spacing. We then derive analytic expressions for the speed of the advancing wave front in the self-oscillatory case and compare them to numerical results. The theoretical results are illustrated by computed solutions of two similar models for calcium wave propagation.
\end{abstract}

Key words. calcium waves, calcium oscillations, excitable systems, periodic medium, traveling waves, periodic plane waves

AMS subject classifications. 35K57, 92C05

PII. S0036139995286035

1. Introduction. Traveling waves of increased intracellular free calcium concentration have been observed in a wide variety of cell types [3, 20,21]. Since $\mathrm{Ca}^{2+}$ is an important intracellular second messenger it is likely that a propagating calcium wave, moving through an individual cell or across a group of cells, serves to coordinate the response of an entire cell, or group of cells, to a local event.

There is thus a great deal of interest in the mechanisms that underlie the propagation of these intracellular and intercellular $\mathrm{Ca}^{2+}$ waves, and a number of models have been proposed [27, 28]. Although the models differ in some respects, most have one property in common; they assume that the wave is propagating through a homogeneous medium. For instance, a recent model for $\mathrm{Ca}^{2+}$ wave propagation in Xenopus oocytes [2] is based on the assumption that the wave is propagated by the diffusion of $\mathrm{Ca}^{2+}$ between inositol 1,4,5-trisphosphate $\left(\mathrm{IP}_{3}\right)$ receptors located on the endoplasmic reticulum (ER). Agonist stimulation of the oocyte results in the production of $\mathrm{IP}_{3}$ which binds to the $\mathrm{IP}_{3}$ receptors and releases $\mathrm{Ca}^{2+}$ from the ER. However, the $\mathrm{IP}_{3}$ receptors are modulated by the cytoplasmic $\mathrm{Ca}^{2+}$ concentration in a complex manner; $\mathrm{Ca}^{2+}$ activates the receptor on a fast time scale but inactivates the receptor on a slower time scale. The interaction of the positive and negative feedback on the receptor can result in $\mathrm{Ca}^{2+}$ oscillations and traveling waves. Although it is well known that $\mathrm{IP}_{3}$ receptors are not distributed uniformly and continuously through the ER, this fact is ignored in the model, which considers the $\mathrm{IP}_{3}$ receptors as "smeared out" in space. In many situations this common assumption will not cause significant errors.

* Received by the editors May 17, 1995; accepted for publication (in revised form) November 20, 1995.

http://www.siam.org/journals/siap/57-1/28603.html

${ }^{\dagger}$ Department of Mathematics and Statistics, University of Canterbury, Private Bag 4800, Christchurch, New Zealand (j.sneyd@math.canterbury.ac.nz). The research of this author was supported by the University of Canterbury, New Zealand, and the Marsden Fund of the Royal Society of New Zealand.

$\ddagger$ Nonlinear Systems Laboratory, Mathematics Institute, University of Warwick, Coventry CV4 7AL, UK (jas@maths.warwick.ac.uk). The research of this author was supported by grants from the Nuffield Foundation and the Royal Society of London. 
If the receptors are dense compared with the size of the domain under consideration then the continuum approximation is valid. Unfortunately, it is not so clear that this approximation is always valid in the modeling of $\mathrm{Ca}^{2+}$ waves.

Thus, it is important to determine how the spacing of receptors (or groups of receptors) affects the existence and speed of traveling waves. The question is complicated by the existence of different types of waves, depending on the concentration of $\mathrm{IP}_{3}$. When $\left[\mathrm{IP}_{3}\right]$ is low, the resting $\mathrm{Ca}^{2+}$ concentration is low also, and $\mathrm{Ca}^{2+}$ waves will not propagate through the cytoplasm. We call this the nonexcitable regime. However, when $\left[\mathrm{IP}_{3}\right]$ is larger, the cell enters the self-oscillatory regime, as positive feedback mechanisms (whether via $\mathrm{Ca}^{2+}$ modulation of the $\mathrm{IP}_{3}$ receptor or by some other mechanism) make the steady state unstable, resulting in oscillations in the $\mathrm{Ca}^{2+}$ concentration and repetitive wave activity. In the self-oscillatory regime, one can observe spiral waves, multiple target patterns, and traveling wave trains. This is typical behavior of a system which has a stable periodic solution. Between the nonexcitable and self-oscillatory regimes is the excitable regime. In this regime a single pulse of $\mathrm{Ca}^{2+}$ or $\mathrm{IP}_{3}$ can initiate a single traveling wave of $\mathrm{Ca}^{2+}$. The wave activity is not repetitive. In summary, as $\left[\mathrm{IP}_{3}\right]$ is increased, the cell moves from a nonexcitable state, to an excitable state, to a self-oscillatory state. This is behavior typical of so-called excitable systems such as the Fitzhugh-Nagumo model or the Hodgkin-Huxley model for the propagation of an action potential in an axon $[6,7]$.

Because waves in the excitable regime are rather different from waves in the selfoscillatory regime, we present an analysis for both types of waves. The first part of the paper studies waves in the excitable regime. We start by considering the simplest possible model for wave propagation in an excitable system. Although of limited biological applicability, the model lends insight into the behavior of more complex models. We then study the behavior of isolated waves in a more general reactiondiffusion model. The second part is concerned with waves in the self-oscillatory region. At each stage, we apply our results to models of $\mathrm{Ca}^{2+}$ wave propagation.

Previously, Dupont and Goldbeter [5] and Mironov [15] performed numerical studies on the effects of discrete receptor spacing on wave propagation. Here, we concentrate on analytical results that are applicable to a wide range of models of $\mathrm{Ca}^{2+}$ wave propagation. A number of other authors have treated the problem of wave block in excitable systems (see, for instance, $[10,12,18]$ ), but mostly these have studied wave block by a change in geometry rather than by inhomogeneities in the excitable medium. Some analytic results for varying or periodic diffusion coefficients are given in $[9,30]$.

\section{Waves in the excitable regime.}

2.1. The simplest model. The simplest model for wave propagation in an excitable system is [14]

$$
u_{t}=D u_{x x}+f(u),
$$

where

$$
f(u)= \begin{cases}-u, & 0 \leq u \leq \alpha, \\ 1-u, & \alpha<u \leq 1 .\end{cases}
$$

The variable $u$ is usually taken as denoting the concentration of the species ( $U$ say) that is propagating the wave. This simple model (the piecewise linear FitzhughNagumo model) has been widely studied and it is well known that it has a traveling wave solution of the form shown in Fig. 1 [19]. Note that the reaction term, $f(u)$, has 


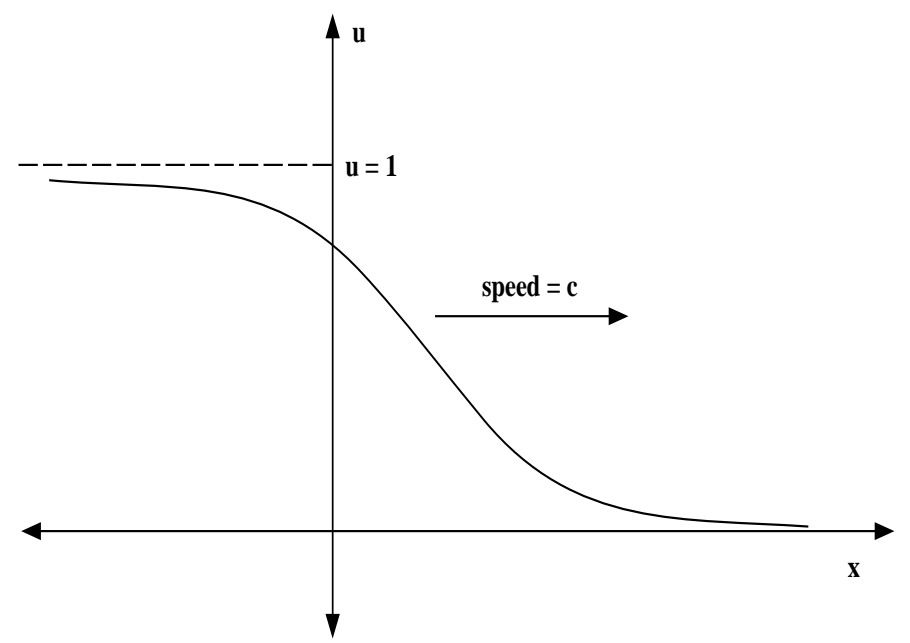

FIG. 1. Schematic diagram of the form of the traveling wave in the piecewise linear FitzhughNagumo equation.

a discontinuity at $x=\alpha$; if $u>\alpha$ the reaction term will drive $u$ toward 1 , while if $u<\alpha$ the reaction term will drive $u$ to 0 . Thus, $\alpha$ acts as a threshold. Throughout most of this paper we shall scale the space variable, $x$, by $\sqrt{D}$ so that $D$ no longer appears explicitly.

In this model, excitability arises due to the properties of the reaction term, $f$, and it is generally assumed that $f$ is given by (2) along the entire $x$-axis. This is equivalent to assuming that the reaction terms that lead to the release of $U$ are homogeneously distributed. However, in many biological situations this may not be the case. Regions where $f$ is given by (2) (active regions) may be separated by regions where there is no release of $U$ or the kinetics are different (passive regions, or gaps).

2.2. Wave propagation across a gap. To investigate how a wave will propagate across a passive region, we set $f=0$ on the interval $[L, L+w]$, as shown in Fig. 2. This interval where the wave is not being actively propagated shall be called the gap. The $x$-axis is thus divided into three regions: region I, $(-\infty, L)$; region II, $[L, L+w]$; and region III, $(L+w, \infty)$.

A traveling wave propagating along the $x$-axis from left to right will cross the gap (and continue to $+\infty$ ) if and only if, at steady state, $u(L+w)>\alpha$ (cf. Fig. 2 ). This is because $\alpha$ is the point of discontinuity (i.e., the threshold) of the reaction term. As soon as $u$ gets above $\alpha$ at the right edge of the gap, $f(u)$ will be positive there, initiating an autocatalytic release of $u$ that will initiate wave propagation on the right-hand side of the gap.

Let $u_{I}, u_{I I}$, and $u_{I I I}$ denote the solutions to (1) in regions I, II, and III, respectively. The steady state equations are

$$
\begin{aligned}
u_{I}^{\prime \prime} & =-\left(1-u_{I}\right), \\
u_{I I}^{\prime \prime} & =0, \\
u_{I I I}^{\prime \prime} & =u_{I I I},
\end{aligned}
$$

and thus

$$
u_{I}=A e^{x}+1
$$




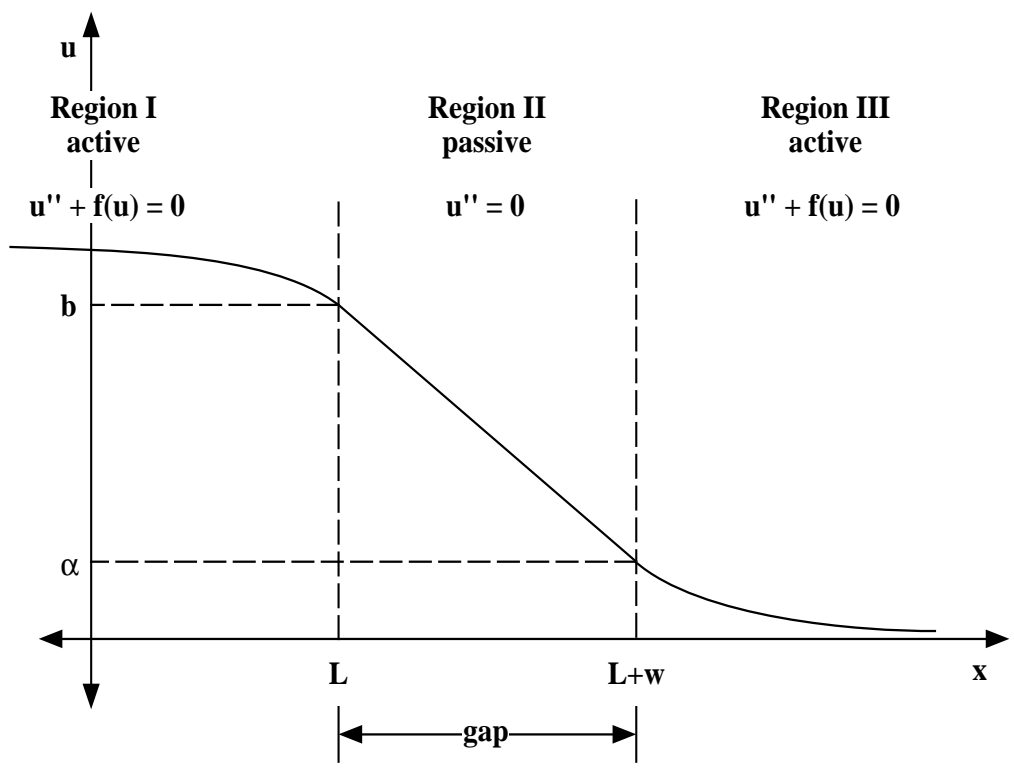

FIG. 2. Illustration of active and passive regions. An active region is where the reaction term, $f(u)$, is nonzero, while a passive region is where $f=0$. Thus, $u$ can move across the gap by passive diffusion only.

$$
\begin{aligned}
u_{I I} & =C x+D, \\
u_{I I I} & =E e^{-x},
\end{aligned}
$$

where we have used boundedness at $\pm \infty$ and where $A, C, D$, and $E$ are constants to be determined. Constraining the steady state solution to be continuous with a continuous derivative at $L$ and $L+w$ gives the constraint equations

$$
\begin{aligned}
A e^{L}+1 & =C L+D, \\
C(L+w)+D & =E e^{-(L+w)}, \\
A e^{L} & =C, \\
C & =-E e^{-(L+w)} .
\end{aligned}
$$

Recalling that $u(L+w)=\alpha$ defines the critical gap width, we can then solve for $w$ to get

$$
w=\frac{1}{\alpha}-2 .
$$

As expected, the critical gap width is independent of $L$ and is a decreasing function of $\alpha$, as one would intuitively expect. As $\alpha$ increases, (1) becomes less excitable, i.e., a greater stimulus is needed to drive the system above threshold, and hence the critical gap width will decrease. Note that $\alpha$ must be less than 0.5 in order for a traveling wave to exist, and therefore the critical gap width is positive. Numerical simulations of these results are shown in Fig. 3.

2.3. A more general model. Now suppose that the wave is governed by the equation

$$
u_{t}=u_{x x}+f(u),
$$



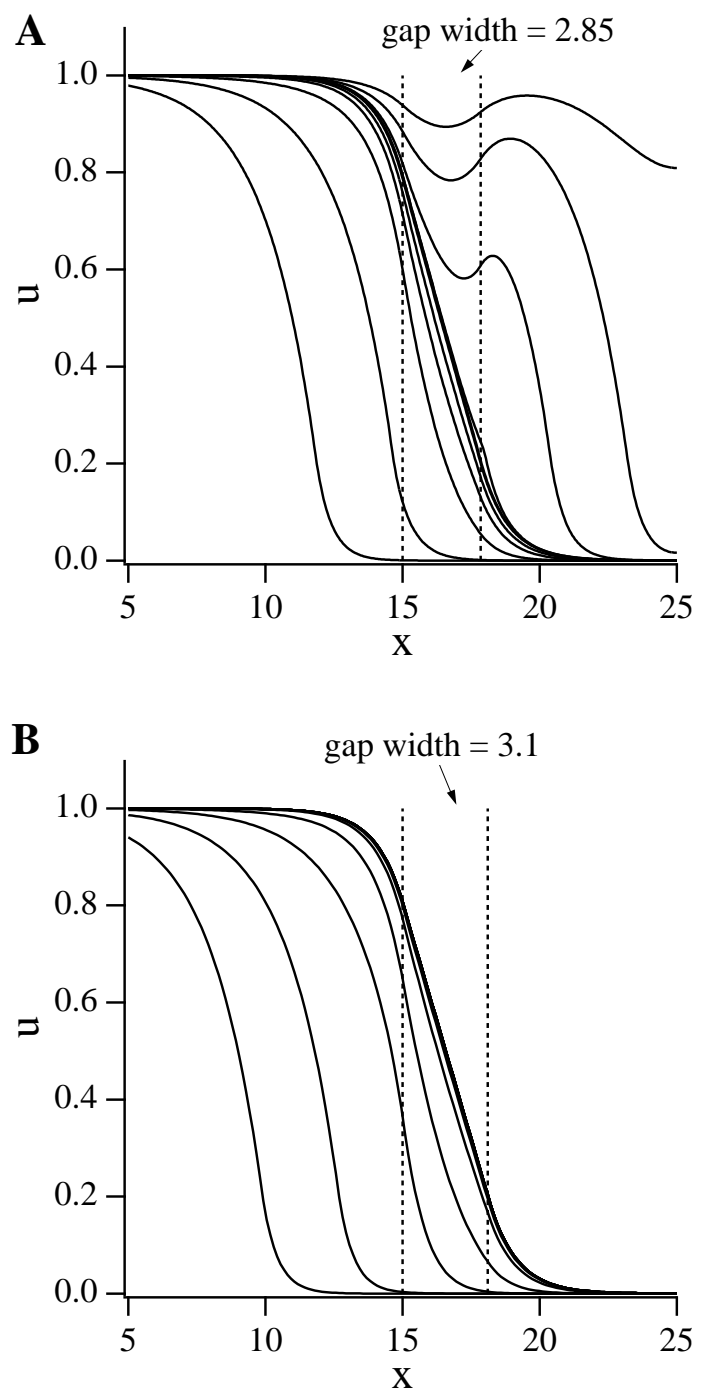

FIG. 3. Numerical solutions to (1), with $\alpha=0.2$. The critical width is thus 3 . (A) When the gap is small enough the wave slows down at the gap, but eventually crosses it. (B) When the gap is wider than the critical width, the wave is not able to cross the gap. The steady solution in the gap is then just a straight line connecting the solutions on either side of the gap.

where $f(u)$ takes the general nonlinear form shown in Fig. 4(A). Equation (14) has two stable steady states at $u=0$ and $u=u_{1}$, and, as before, the saddle point at $u=\alpha$ acts as a threshold.

Set up the domain as before, with a gap (i.e., $f=0$ ) on the interval $[L, L+w]$ and active kinetics on $(-\infty, L)$ and $(L+w, \infty)$. At steady state $u^{\prime \prime}=-f(u)$ and thus

$$
\frac{1}{2} \frac{d}{d x}\left(u^{\prime}\right)^{2}=\frac{d}{d x} G(u)
$$

where

$$
G(u)=-\int_{u_{0}}^{u} f(v) d v
$$



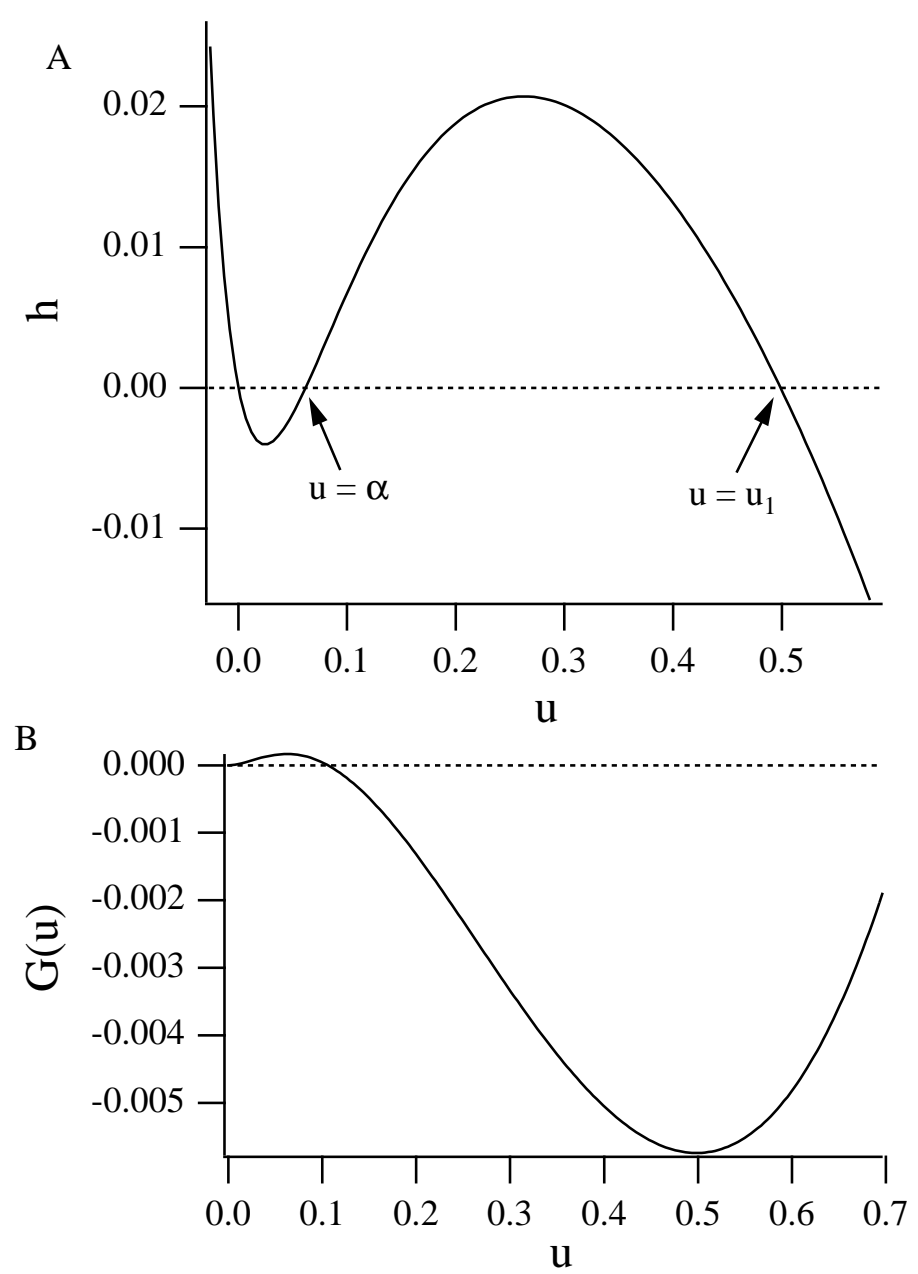

FIG. 4. (A) Nullcline $(d u / d t=0)$ of the Li-Rinzel model for $\left[\mathrm{IP}_{3}\right]=\mu=0.3 \mu \mathrm{M}$. It is an $N$-shaped curve typical of excitable systems. (B) The function $G(u)$ calculated from (16) for the same parameter values as $(\mathrm{A})$.

A sketch of $G$ is given in Fig. 4(B). It follows that for any $x_{0}$ and $x_{1}$,

$$
\left.\frac{1}{2}\left(u^{\prime}\right)^{2}\right|_{x=x_{0}} ^{x=x_{1}}=\left.G(u)\right|_{x=x_{0}} ^{x=x_{1}} .
$$

Let $x_{0}=-\infty$ and $x_{1}=x<L$ to get

$$
\frac{1}{2}\left(u^{\prime}\right)^{2}=G(u)-G\left(u_{1}\right),
$$

where we have used the fact that, behind the wave front, $u=u_{1}$. Note that $u>\alpha$ on $(-\infty, L)$. Similarly, on $(L+w, \infty)$ we have

$$
\frac{1}{2}\left(u^{\prime}\right)^{2}=G(u)-G\left(u_{0}\right)=G(u),
$$

where we use the fact that, in front of the wave, $u=u_{0}$. 
On the interval $[L, L+w]$, a steady state solution for $u$ must be a straight line, and thus matching the slopes of $u$ at $L$ and $L+w$ gives

$$
\frac{1}{2}\left(\frac{u(L)-u(L+w)}{w}\right)^{2}=G[u(L)]-G\left(u_{1}\right)=G[u(L+w)] .
$$

This equation has a solution for values of $u(L+w)$ either side of the threshold value $\alpha$. However, we expect intuitively that the steady state can only be stable when $u(L+w)<\alpha$, and this is confirmed by numerical simulation. Thus the critical gap width above which the wave cannot propagate is given by $u(L+w)=\alpha$; substituting this into (20) and eliminating $u(L)$ gives an equation for $w$ with a unique solution.

2.3.1. Inclusion of degradation in the gap. In many situations the wave variable, $u$, does not simply diffuse across the gap but is degraded or removed as well. For instance, the regions of active kinetics could refer to regions where there are many $\mathrm{IP}_{3}$ receptors, but in between these regions $\mathrm{Ca}^{2+}$ does not simply diffuse but is pumped back into the ER. Therefore, a more realistic model could include the effect of the removal of $u$ in the gap.

We make the simplest assumption that the removal of $u$ in the gap is a first-order process, i.e.,

$$
u_{t}=u_{x x}-\delta^{2} u
$$

and, thus, in the gap

$$
u^{\prime \prime}=\delta^{2} u
$$

at steady state for some constant $\delta$. A similar analysis to the above shows that the critical gap width, $w$, and $u(L)$ are given by

$$
\begin{aligned}
\alpha-u(L) \cosh (\delta w) & =-\frac{1}{\delta} \sinh (\delta w) \sqrt{2\left[G[u(L)]-G\left(u_{1}\right)\right]}, \\
\alpha \cosh (\delta w)-u(L) & =-\frac{1}{\delta} \sinh (\delta w) \sqrt{2 G(\alpha)} .
\end{aligned}
$$

2.4. Application to calcium waves. Li and Rinzel [13] have proposed a model for $\mathrm{Ca}^{2+}$ oscillations based on a reduction of a previous model by DeYoung and Keizer [4]. The model includes terms that describe the $\mathrm{Ca}^{2+}$ current through the $\mathrm{IP}_{3}$ receptor, modulation of the receptor current by $\mathrm{IP}_{3}$ and $\mathrm{Ca}^{2+}$, leak of $\mathrm{Ca}^{2+}$ out of the ER, and reuptake of $\mathrm{Ca}^{2+}$ by an ATPase pump. The model equations take the form

$$
\begin{aligned}
\frac{d u}{d t} & =f(u, h ; \mu), \\
\tau_{h}(u ; \mu) \frac{d h}{d t} & =h_{\infty}(u ; \mu)-h,
\end{aligned}
$$

where $u$ denotes $\left[\mathrm{Ca}^{2+}\right]$ and $\mu$, which denotes $\left[\mathrm{IP}_{3}\right]$, is treated as a bifurcation parameter. The complete model equations, and the parameters we used for computations, are given in the appendix. The nullcline $f(u, h ; \mu=0.3)=0$ is graphed in Fig. 4(A), where for simplicity the steady state has been shifted to the origin. It is an N-shaped curve typical of models of excitable systems. Assuming $h$ to be a slow variable and incorporation of $\mathrm{Ca}^{2+}$ diffusion in one space dimension give

$$
u_{t}=D_{c} u_{x x}+f\left(u, h_{0} ; \mu\right)
$$




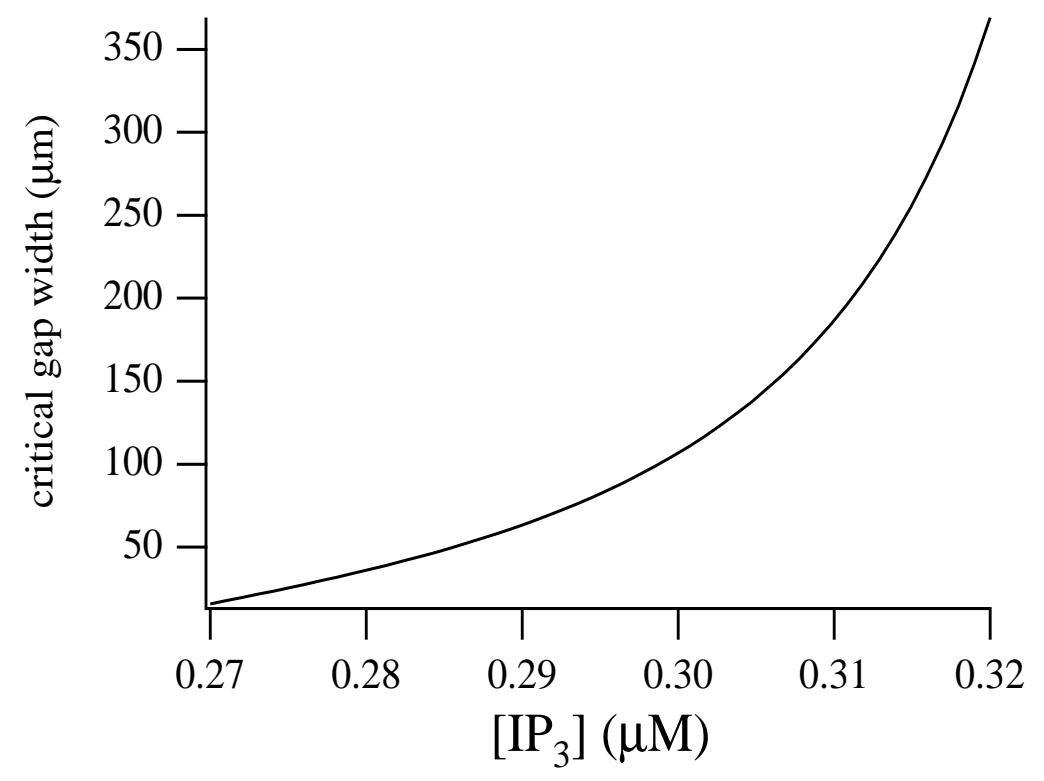

FIG. 5. Critical gap width as a function of [ $\left.\mathrm{IP}_{3}\right]$ for the Li-Rinzel model [13]. As [ $\left.\mathrm{IP}_{3}\right]$ increases, the critical width becomes large since the threshold, $\alpha$, is decreasing to zero, but the time taken for the wave to cross the gap also tends to infinity (computations not shown).

where $D_{c}$ is the diffusion coefficient of $\mathrm{Ca}^{2+}$ and $h_{0}$ is the steady state of $h$. Note that since $h$ is a slow variable, $h$ is constant over the wave front. We implicitly assume that $\mathrm{Ca}^{2+}$ buffers may be approximated by an apparent diffusion coefficient, an assumption which has many dangers [26, 29], but a full discussion of the complications caused by buffers is outside the scope of this paper. $G(u)$, defined by (16), is plotted in Fig. 4(B). In Fig. 5 we plot the critical width as a function of $\mu$. We used $D_{c}=25 \mu \mathrm{m}^{2} \mathrm{~s}^{-1}$, a value obtained experimentally in Xenopus cytoplasm [1]. In general, since the diffusion coefficient merely sets the length scale, it is sufficient to calculate the critical width for the case $D_{c}=1$ and then rescale appropriately. The critical width has a very sensitive dependence on $\left[\mathrm{IP}_{3}\right]$ and is less than $20 \mu \mathrm{m}$ for low $\mathrm{IP}_{3}$. This is consistent with the results of Parker and Yao [17], who observed punctate release of $\mathrm{Ca}^{2+}$ from isolated "hot spots" in Xenopus at concentrations of $\mathrm{IP}_{3}$ that were too low to initiate propagating waves. Nevertheless, the quantitative model predictions should be treated with caution, as the calculation of the critical width does not take receptor inactivation into account. Relaxing the assumption that $h$ is a slow variable will give more accurate predictions, but as yet we have not been able to do this analytically.

3. Waves in the self-oscillatory regime. Consider the reaction-diffusion system

$$
\begin{aligned}
& u_{t}=u_{x x}+M(x) f(u, v), \\
& v_{t}=M(x) g(u, v) .
\end{aligned}
$$

We assume the medium is inhomogeneous and thus the reaction terms $f$ and $g$ are modulated by $M(x)$. In order to make the problem tractable, we shall assume that $M$ is periodic and piecewise constant and divides the spatial domain into alternating active and passive regions (Fig. 6). Each active region is of width $L_{1}$, and each 


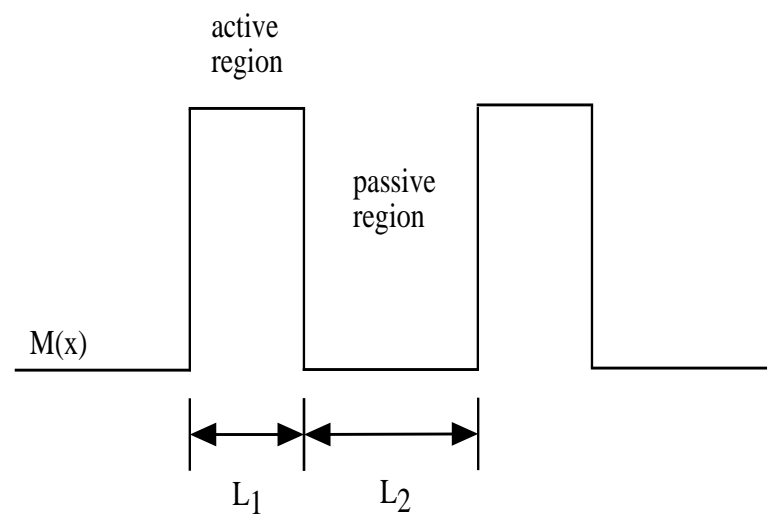

FIG. 6. Schematic diagram of the function $M(x)$ which controls the spatial inhomogeneity in (28) and (29). $M$ divides the spatial domain into alternating active and passive regions. Usually, we shall assume that $M=0$ in the passive regions (i.e., that $b_{1}, \ldots, b_{4}=0$ ), but that is not necessary for the analysis.

passive region is of width $L_{2}$. We wish to investigate how the existence and speed of propagating waves depend on $L_{1}$ and $L_{2}$. Our analysis is similar to that of Shigesada, Kawasaki, and Teramoto [25], who studied the propagation of waves in the Fisher equation in an inhomogeneous medium. We assume that (28) and (29) have a unique, spatially homogeneous steady state $\left(u_{0}, v_{0}\right)$. We assume further that, in the absence of diffusion, the steady state is unstable via growing oscillations and that a stable limit cycle exists. Although this may seem unduly restrictive, it is the case in the application of interest to us here. Typically, in models of $\mathrm{Ca}^{2+}$ wave propagation, the space-independent model equations become unstable via a Hopf bifurcation, and the above conditions are satisfied. The analysis in the more general case is similar to that given here and is omitted.

3.1. Stability of the steady state. Linearizing (28) and (29) about the steady state, which we may assume to be the origin without loss of generality, gives the linear system

$$
\begin{aligned}
& u_{t}=u_{x x}+M(x)\left(f_{u} u+f_{v} v\right), \\
& v_{t}=M(x)\left(g_{u} u+g_{v} v\right),
\end{aligned}
$$

where the partial derivatives of $f$ and $g$ are evaluated at $(u, v)=(0,0)$. Usually, stability is determined by looking for solutions of the form $\exp (\lambda t+i \omega x)$ and then determining $\lambda$ as a function of $\omega$ (the dispersion curve). However, here we have the added complication of the spatial periodicity in addition to the periodicity of $\exp (i \omega x)$. In other words, we wish to consider the stability of the spatially periodic system to perturbations of all wave numbers. Hence, we look for solutions of the form

$$
\begin{aligned}
& u=\phi(x) e^{\lambda t+i \omega x}, \\
& v=\theta(x) e^{\lambda t+i \omega x},
\end{aligned}
$$

where $\phi$ and $\theta$ are periodic and $\lambda$ and $\omega$ are real, which gives

$$
\phi^{\prime \prime}+2 i \omega \phi^{\prime}-\left\{\frac{\lambda^{2}+\lambda\left(\omega^{2}-M f_{u}-M g_{v}\right)+M^{2}\left(f_{u} g_{v}-f_{v} g_{u}\right)-M g_{v} \omega^{2}}{\left(\lambda-M g_{v}\right)}\right\} \phi=0 .
$$


Let

$$
M(x)\left(\begin{array}{ll}
f_{u} & f_{v} \\
g_{u} & g_{v}
\end{array}\right)= \begin{cases}\left(\begin{array}{ll}
a_{1} & a_{2} \\
a_{3} & a_{4}
\end{array}\right), & \text { active region, } \\
\left(\begin{array}{ll}
b_{1} & b_{2} \\
b_{3} & b_{4}
\end{array}\right), & \text { passive region, }\end{cases}
$$

where $a_{1}, \ldots, a_{4}$ and $b_{1}, \ldots, b_{4}$ are constants. Then, the condition for the existence of a $C^{1}$ periodic solution of $(34)$ is

$$
\frac{\alpha^{2}+\beta^{2}}{2 \alpha \beta} \sinh \left(\alpha L_{1}\right) \sinh \left(\beta L_{2}\right)+\cosh \left(\alpha L_{1}\right) \cosh \left(\beta L_{2}\right)=\cos \left[\omega\left(L_{1}+L_{2}\right)\right]
$$

where

$$
\begin{aligned}
& \alpha=\sqrt{\frac{\lambda^{2}-\lambda\left(a_{1}+a_{4}\right)+\left(a_{1} a_{4}-a_{2} a_{3}\right)}{\left(\lambda-a_{4}\right)}}, \\
& \beta=\sqrt{\frac{\lambda^{2}-\lambda\left(b_{1}+b_{4}\right)+\left(b_{1} b_{4}-b_{2} b_{3}\right)}{\left(\lambda-b_{4}\right)}} .
\end{aligned}
$$

For a fixed $L_{1}$ and $L_{2},(36)$ defines $\lambda$ as a function of $\omega$. The maximum of $\lambda(\omega)$ defines the fastest growing mode; if $\lambda>0$ at this maximum, the steady state is unstable, while if $\lambda<0$ at the maximum, the steady state is stable. Thus, in principle it is possible to calculate the boundary between stability and instability for a particular model. In the application to $\mathrm{Ca}^{2+}$ waves we are most interested in the case when $L_{1}$ and $L_{2}$ are small, as in general they are both considerably smaller than the diffusion coefficient of $\mathrm{Ca}^{2+}$. So, let $L_{1}=\epsilon \tilde{\tilde{L}}_{1}, L_{2}=\epsilon \tilde{L}_{2}$, where $\epsilon \ll 1$. Then, to leading order in $\epsilon,(36)$ becomes

and thus

$$
\frac{1}{2}\left(\alpha^{2}+\beta^{2}\right) \tilde{L}_{1} \tilde{L}_{2}+\frac{1}{2} \alpha^{2} \tilde{L}_{1}^{2}+\frac{1}{2} \beta^{2} \tilde{L}_{2}^{2}=-\frac{\omega^{2}}{2}\left(\tilde{L}_{1}+\tilde{L}_{2}\right)^{2}
$$

$$
\alpha^{2} \tilde{L}_{1}+\beta^{2} \tilde{L}_{2}=-\omega^{2}\left(\tilde{L}_{1}+\tilde{L}_{2}\right) .
$$

Substituting in the expressions for $\alpha$ and $\beta$ and assuming for convenience that $b_{1}, \ldots, b_{4}$ are zero give

$$
\left(\tilde{L}_{1}+\tilde{L}_{2}\right) \lambda^{2}-\left\{\left(a_{1}+a_{4}\right) \tilde{L}_{1}+a_{4} \tilde{L}_{2}-\omega^{2}\left(\tilde{L}_{1}+\tilde{L}_{2}\right)\right\} \lambda+\tilde{L}_{1}\left(a_{1} a_{4}-a_{2} a_{3}\right)-\omega^{2} a_{4}\left(\tilde{L}_{1}+\tilde{L}_{2}\right)=0 .
$$

In order for the solutions to this equation to have negative real part for all $\omega$, we must have that

$$
\tilde{L}_{1}\left(a_{1}+a_{4}\right)+a_{4} \tilde{L}_{2}<0
$$

and thus

$$
\tilde{L}_{1}<\frac{-a_{4}}{a_{1}+a_{4}} \tilde{L}_{2}
$$

Since $a_{1}+a_{4}>0$ (because by assumption the homogeneous steady state is unstable via growing oscillations), this requires $a_{4}<0$, which is the case for the models of $\mathrm{Ca}^{2+}$ waves we discuss here.

When $L_{2}=0,(36)$ reduces to

$$
\lambda^{2}+\lambda\left(\omega^{2}-a_{1}-a_{4}\right)+a_{1} a_{4}-a_{2} a_{3}-a_{4} \omega^{2}=0,
$$

which is the usual dispersion equation for a homogeneous domain. 


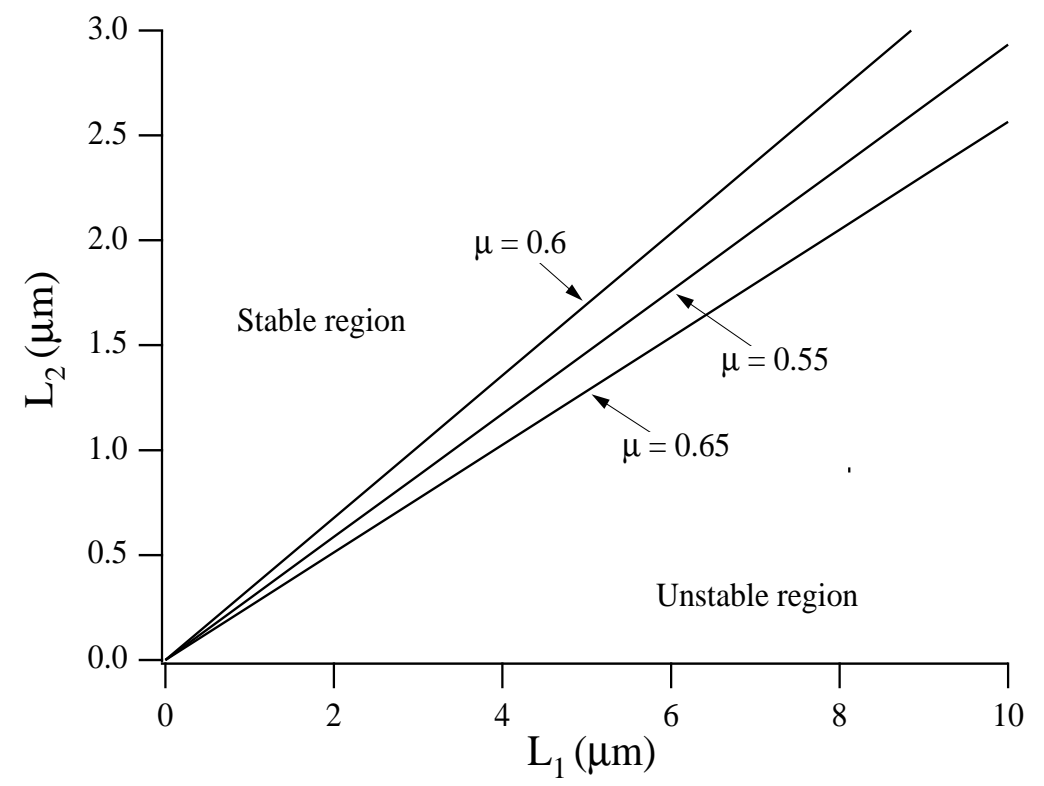

Fig. 7. The boundary between instability and stability in $L_{1}, L_{2}$ space calculated from (43) for the Atri et al. model [2]. The boundary is shown for three different values of $\mu$ corresponding to three different values of $\left[\mathrm{IP}_{3}\right]$. As explained in the text, the slope of the boundary line is not a monotonic function of $\mu$. This stability boundary has been confirmed numerically (computations not shown).

3.2. Another model for calcium waves. We shall illustrate the results on periodic plane waves with the model of Atri et al. [2]. The model equations have the same form as those of $\mathrm{Li}$ and Rinzel, but the functional forms of $f$ and $h_{\infty}$ are different. Thus, the model is easily written in the form of (28) and (29), with $v$ replacing $h$ and $g$ replacing $\left(h_{\infty}-h\right) / \tau_{h}$. Again, the full equations and parameters are given in the appendix. As $\mu$ increases, the model (often but not always) exhibits oscillations via a Hopf bifurcation, and as $\mu$ increases still further, the oscillations disappear again via another Hopf bifurcation. In general, between the two Hopf bifurcations, the steady state of the temporal equations is unstable via growing oscillations. In Fig. 7 we show a number of lines of the form $L_{1}=-a_{4} L_{2} /\left(a_{1}+a_{4}\right)$ for different values of $\mu$ (corresponding to different values of $\left[\mathrm{IP}_{3}\right]$ ), each line giving the boundary between instability and stability. Note that the slope of the boundary line is not a monotonic function of $\mu$ : since $-a_{4} /\left(a_{1}+a_{4}\right) \longrightarrow \infty$ at the Hopf bifurcations (i.e., when $\left.a_{1}+a_{4}=0\right)$ it must have a (positive) minimum value. It thus follows that the boundary line has a maximum slope as $\mu$ varies, and thus if $L_{2}$ is large enough and for a fixed value of $L_{1}$, the steady state will be stable for all values of $\mu$. One surprising consequence of this is that the model predicts that the addition of further $\mathrm{IP}_{3}$ can sometimes stabilize an unstable steady state and lead to the disappearance of waves, even though the underlying steady state is still unstable. This is actually consistent with experimentally observed waves, where the addition of too much $\mathrm{IP}_{3}$ can eliminate wave activity. Unfortunately, in the experimental situation it is not easy to tell whether the underlying steady state is stable or not, and therefore this model prediction will be difficult if not impossible to verify. Numerical solution of the differential equations confirms this stability boundary when $L_{1}$ and $L_{2}$ are small.

3.2.1. Numerical methods. Numerical solutions are obtained in the following way. First, $\mu$ is chosen so that the model has a stable limit cycle. Heaviside initial 
conditions are used for $c$. Thus, at $t=0, c$ on the left portion of the domain (usually the 10 leftmost spatial grid points) is raised above steady state, initiating oscillations in the leftmost region of the domain. If stable periodic waves exist, these oscillations gradually invade the entire domain. However, if the periodic wave is unstable, the oscillations gradually disappear. By looking at the existence of periodic waves for different (small) values of $L_{1}$ and $L_{2}$, we were able to confirm the predicted stability boundary. The differential equations were solved using an implicit time-stepping scheme and central space differences.

3.2.2. Periodic waves and the wave front. It is crucial to note that, in the numerical solutions, two very different types of waves appear. We distinguish them by the terms periodic wave and wave front. This is most easily illustrated by reference to Fig. 12, which shows the situation a short time after $t=0$ for the case $L_{2}=0$ and when Heaviside initial conditions for $c$ were used, as described above. Periodic waves exist for this value of $L_{2}$ because the domain is homogeneous with oscillatory kinetics. The periodic wave can be clearly seen as a series of white bands sloping up from left to right. These correspond to a periodic wave moving from right to left across the domain. However, the periodic wave is not the same as the wave front. As time increases, each successive peak of the periodic wave is initiated from a region that is moving slowly from left to right. This we call the wave front of the periodic wave; its approximate position is marked by a line in Fig. 12. When $t$ is large enough, the wave front will have moved across the entire domain, leaving only the periodic wave in its wake. Note that the wave front is moving from left to right with a speed that is much less than that of the periodic wave which is moving in the opposite direction. The analysis in the present paper is confined to studying the speed of the wave front, not the speed of the subsequent periodic wave. It is also important to note that the wave front, which we define as the place where $c=0.05 \mu \mathrm{M}$, does not move across the domain monotonically (cf. Fig. 10). This is caused by the oscillatory nature of the kinetics. Nevertheless, the average wave front speed may still be defined as the slope of the best fit line through the wave front.

Extensive numerical simulations with different initial conditions and values of $\mu$ show that the periodic wave can travel with a wide range of speeds in either direction. In some cases, as in Fig. 12, it starts at the wave front, travels backward across the domain, and is absorbed by the left-hand boundary. In other cases, the periodic wave is initiated at the left-hand boundary and travels from left to right until it hits the wave front and disappears. Sherratt $[22,24,23]$ discusses this in detail for periodic waves in $\lambda-\omega$ systems. A useful interpretation of the wave front speed is the speed at which a periodic wave can "invade" a domain. However, the actual speed and direction of the periodic wave can be very different from the speed at which that periodic wave invades a domain.

3.3. The dispersion curve. The oscillatory nature of the kinetics means that wave solutions of (28) and (29) are not simple transition waves, as in the excitable case. Rather, they have the form of a moving wave front with spatiotemporal oscillations behind the front (as described above). We focus on the movement of the leading wave front, which we study using the linearized equations (30) and (31). The linear analysis will apply to the front of the nonlinear wave and will provide an approximate estimate for the nonlinear wave front speed. Numerical results confirm the approximate accuracy of estimates from the linear theory. We first consider the spatially homogeneous case and use this as a guide to the analysis of the spatially heterogeneous case. 
We emphasize that in both the homogeneous and the heterogeneous cases, our analysis is a local one only and therefore applies only to the movement of the wave front. The speed of the periodic waves behind the wave front cannot be treated by our methods. In fact, we have as yet been unable to determine the speed of the periodic waves as a function of $L_{1}$ and $L_{2}$. This requires a nonlinear analysis which is considerably more difficult than that presented here.

3.3.1. Spatially homogeneous case. It is helpful to consider first the slightly different case where $u$ and $v$ have equal diffusion coefficients, i.e.,

$$
\begin{aligned}
& u_{t}=u_{x x}+f_{u} u+f_{v} v, \\
& v_{t}=v_{x x}+g_{u} u+g_{v} v .
\end{aligned}
$$

By a change of coordinates these equations can be written in the form

$$
\begin{aligned}
& u_{t}=u_{x x}+\alpha u+\omega v, \\
& v_{t}=v_{x x}-\omega u+\alpha v .
\end{aligned}
$$

Define new coordinates by $u=r \cos \theta, v=r \sin \theta$ and convert to $r, \theta$ coordinates to get

$$
\begin{aligned}
& r_{t}=r_{x x}-r \theta_{x}^{2}+\alpha r, \\
& \theta_{t}=\theta_{x x}+2 \frac{r_{x} \theta_{x}}{r}-\omega .
\end{aligned}
$$

Linearizing about the wave front, where $r=\theta_{x}=0$ [23], gives

$$
r_{t}=r_{x x}+\alpha r
$$

Since (51) is the same form as the linearization of Fisher's equation, we merely apply previous results (see, for example, $[8,11,16]$ ) to determine the wave front speed. Let $z=x-c t$, where $c$ is the wave front speed. Then, looking for solutions of the form $r=\exp (-s z)$ gives $c s=s^{2}+\alpha$ and thus $c=s+\alpha / s$. In general, the wave speed depends on the initial conditions. However, for Heaviside initial conditions of the type used here the wave travels with the minimum speed. Since this type of initial condition is the only one used experimentally and is thus the only case of physiological interest, we do not consider any other initial conditions. Thus the observed wave speed, $c_{o}$, is given by $c_{o}=2 \sqrt{\alpha}$.

Unfortunately, this change of variables does not work if $v$ and $u$ have different diffusion coefficients. Previous work on $\lambda-\omega$ systems [22, 24, 23] shows that in front of the wave $\theta$ does not go to zero but increases (i.e., oscillates) according to $\theta=\omega t$. It is reasonable to expect that something similar happens here also. This suggests that the wave front is not only oscillatory in $z$ but also oscillatory in $t$, which motivates the following analysis. Let $D$ be the diffusion coefficient of $v$, i.e.,

$$
\begin{aligned}
& u_{t}=u_{x x}+\alpha u+\omega v, \\
& v_{t}=D v_{x x}-\omega u+\alpha v,
\end{aligned}
$$

and look for solutions of the form $u=A e^{-s z} e^{i \omega t} v=B e^{-s z} e^{i \omega t}$ for some real constants $A$ and $B$ and where $s$ is complex. The solvability condition for $A$ and $B$ turns out to be

$$
\left(\xi-\sigma_{1}\right)\left(\xi-\sigma_{2}\right)=-\omega^{2},
$$


where $\xi=c s, \sigma_{1}=D s^{2}+\alpha-i \omega$, and $\sigma_{2}=s^{2}+\alpha-i \omega$. Letting $s=\gamma+i \delta$ gives

$$
\begin{aligned}
& \phi_{1}(c, \delta)=0, \\
& \phi_{2}(c, \delta)=0,
\end{aligned}
$$

where

$$
\begin{aligned}
\phi_{1}(c, \delta)=\left(c \gamma-D \gamma^{2}+\right. & \left.D \delta^{2}-\alpha\right)\left(c \gamma-\gamma^{2}+\delta^{2}-\alpha\right) \\
- & (c \delta-2 D \gamma \delta+\omega)(c \delta-2 \gamma \delta+\omega)+\omega^{2}, \\
\phi_{2}(c, \delta)=\left(c \gamma-D \gamma^{2}\right. & \left.+D \delta^{2}-\alpha\right)(c \delta-2 \gamma \delta+\omega) \\
& +\left(c \gamma-\gamma^{2}+\delta^{2}-\alpha\right)(c \delta-2 D \gamma \delta+\omega) .
\end{aligned}
$$

For fixed $\gamma$ and $D$, we can plot the curves $\phi_{1}=0, \phi_{2}=0$ to get curves in the $c, \delta$ plane. The intersections of these curves give $c$ and $\delta$ for that value of $\gamma$, and then varying $\gamma$ gives $c$ and $\delta$ as (possibly multivalued) functions of $\gamma$. This is illustrated in Fig. 8. When $D=1$, there are at least three solutions for $c$ and $\delta$, but only the one with $\delta=0$ is of interest, as it corresponds to the solutions found above. Thus, as we vary $\gamma$, we track only that solution branch. Note that when $D=1$ this solution branch gives $c=\gamma+\alpha / \gamma$, as can be obtained directly from (54). Presumably the other branches correspond to unstable waves (or waves moving in the opposite direction), but we have not investigated this.

Although $\delta$ increases and $c$ decreases as $D$ decreases from one, Fig. 8 indicates that they do not change a great deal. Thus motivated, we let $D=1-\sum_{n=1}^{\infty} \epsilon^{n}$ and look for solutions of the form $\delta=\epsilon \delta_{1}+\epsilon^{2} \delta_{2}+O\left(\epsilon^{3}\right), c=\gamma+\alpha / \gamma+\epsilon c_{1}+\epsilon^{2} c_{2}+O\left(\epsilon^{3}\right)$. Note that $0 \leq \epsilon \leq 0.5$ if $0 \leq D \leq 1$. Substituting into (57) and (58) and equating coefficients of powers of $\epsilon$ give $c_{1}=c_{2}=-\gamma / 2$, and thus

$$
\begin{aligned}
c= & \gamma+\frac{\alpha}{\gamma}-\frac{\gamma}{2}\left(\epsilon+\epsilon^{2}\right)+O\left(\epsilon^{3}\right), \\
& \approx \gamma+\frac{\alpha}{\gamma}-\frac{\gamma}{2} \frac{(1-D)(3-2 D)}{(2-D)^{2}},
\end{aligned}
$$

where we have used $\epsilon=(1-D) /(2-D)$. When $D=0, c=5 \gamma / 8+\alpha / \gamma$. That (60) is an excellent approximation can be seen from Fig. 9, where we plot the actual and asymptotic solutions for various $D$ s in the Atri et al. model. Note that because of scaling $D=1$ in (60) is equivalent to $D=D_{c}$ in the Atri model (see Appendix). The curve for $D=0$ is in fact a discontinuous combination of two different solution branches: it is somewhat surprising that this discontinuous combination can be approximated so well by the theoretical prediction.

It is reasonable to assume that for the Heaviside initial conditions that we use the observed wave fronts travel at the lowest possible speed, as in Fisher's equation, and thus the observed wave front speed, $c_{o}$, is given by

$$
c_{o}=\sqrt{2 \alpha\left(2-\frac{(1-D)(3-2 D)}{(2-D)^{2}}\right)} .
$$

This is confirmed by numerical results from the Atri et al. model, summarized in Fig. 10 , where we plot the position of the wave front as a function of time. Oscillations in the wave front (which is defined as the place where $c$ equals some given value, 0.05 in this case) result in a nonmonotonic progression of the wave across the domain, but 
A

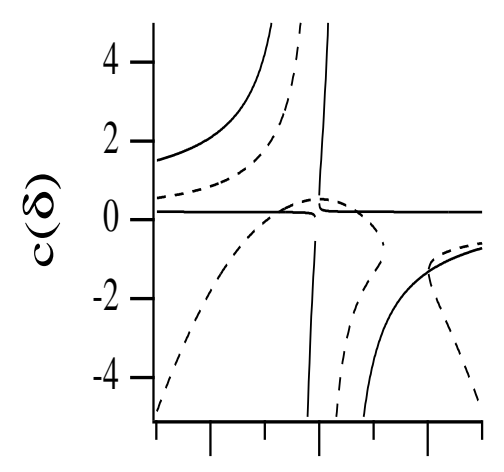

C

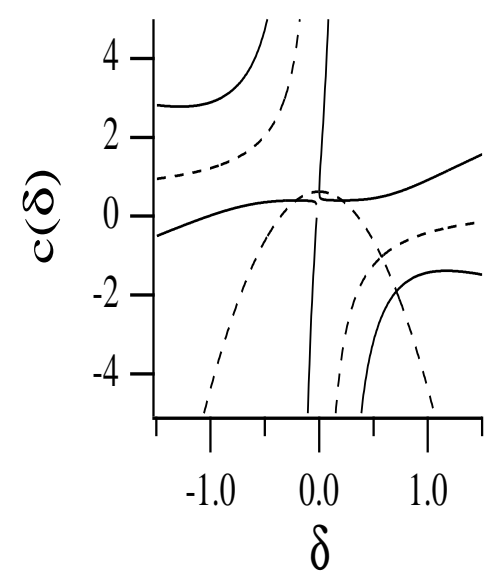

B

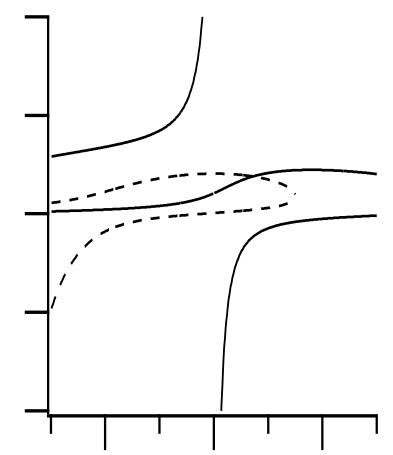

D

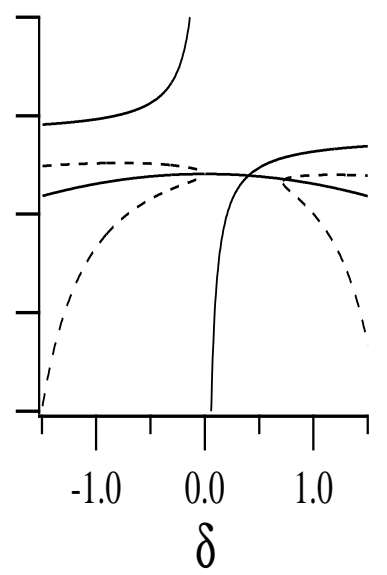

FIG. 8. Plots of $\phi_{1}(c, \delta)=0$ (solid curve) and $\phi_{2}(c, \delta)=0$ (dashed curve) calculated from the Atri et al. model for $\mu=0.7$. For these computations, $x$ was scaled by the square root of the diffusion coefficient of $\mathrm{Ca}^{2+}$, and hence $D_{c}=1$. Each curve is drawn for fixed values of $\gamma$ and $D$, and, as explained in the text, intersections of the solid and dashed curves give $c$ and $\delta$ as multivalued functions of $\gamma$ and $D$. However, only one of those branches appears to correspond to an observed solution. (A) $\gamma=0.2, D=0$. (B) $\gamma=2, D=0$. (C) $\gamma=0.2, D=1$. (D) $\gamma=2, D=1$.

the wave speed can be defined most simply as the slope of the best-fit line through the data points. When $D=D_{c}, c_{o}=2.08 \mu \mathrm{m} / \mathrm{s}$, compared with the predicted value of $2.04 \mu \mathrm{m} / \mathrm{s}$, whereas when $D=0, c_{o}=1.74 \mu \mathrm{m} / \mathrm{s}$, compared with the predicted value of $1.61 \mu \mathrm{m} / \mathrm{s}$.

It is important to note that although the wave front moves across the domain at a speed given approximately by (61), the periodic waves behind the wave front move with a different speed and direction. This has been demonstrated analytically for $\lambda-\omega$ systems [24, 23] and holds true in the general case. The present analysis is of relevance only for the wave front velocity and is not applicable to the velocity of the periodic waves behind the wave front.

3.3.2. Spatially heterogeneous case. We shall consider only the case $D=0$. The analysis is similar for nonzero $D$, only rather more tedious. Motivated by the 


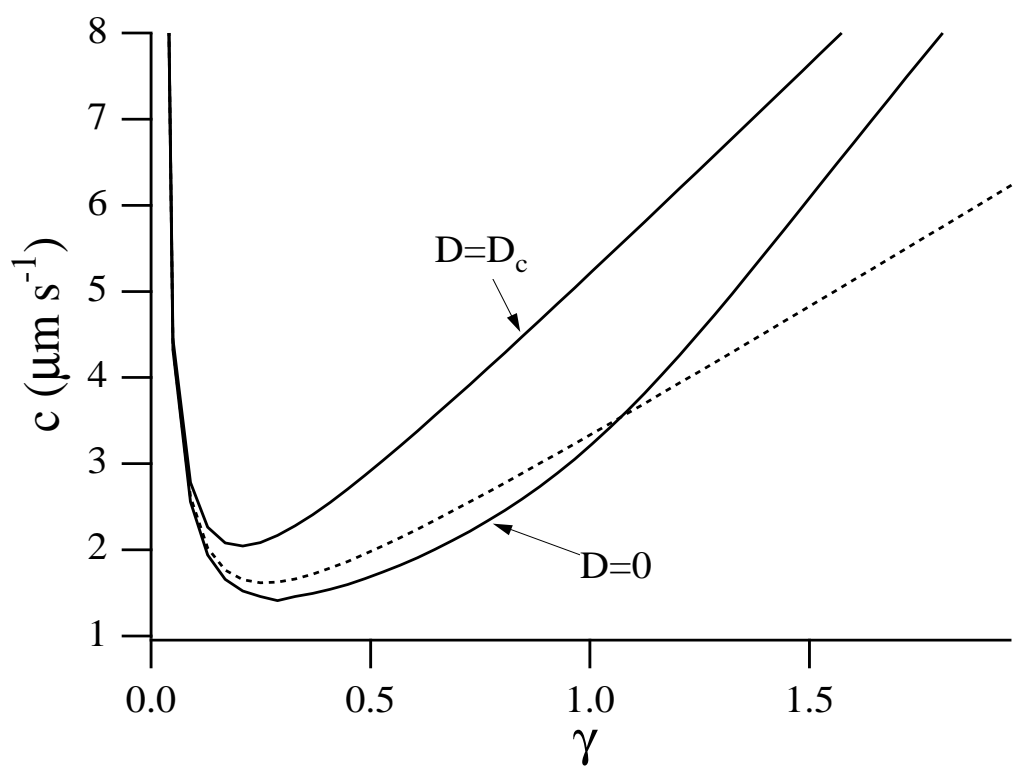

FIG. 9. Wave front speed $c$ as a function of $\gamma$ in the Atri et al. model, calculated from one of the intersection branches in Fig. 8. The dotted line is the analytical approximation (60) to the $D=0$ curve. The branch of intersections corresponding to $D=0$ is a discontinuous combination of two different branches. The minimum of these curves is a good approximation to the wave front speed obtained by numerical solution of the PDE (Fig. 10).

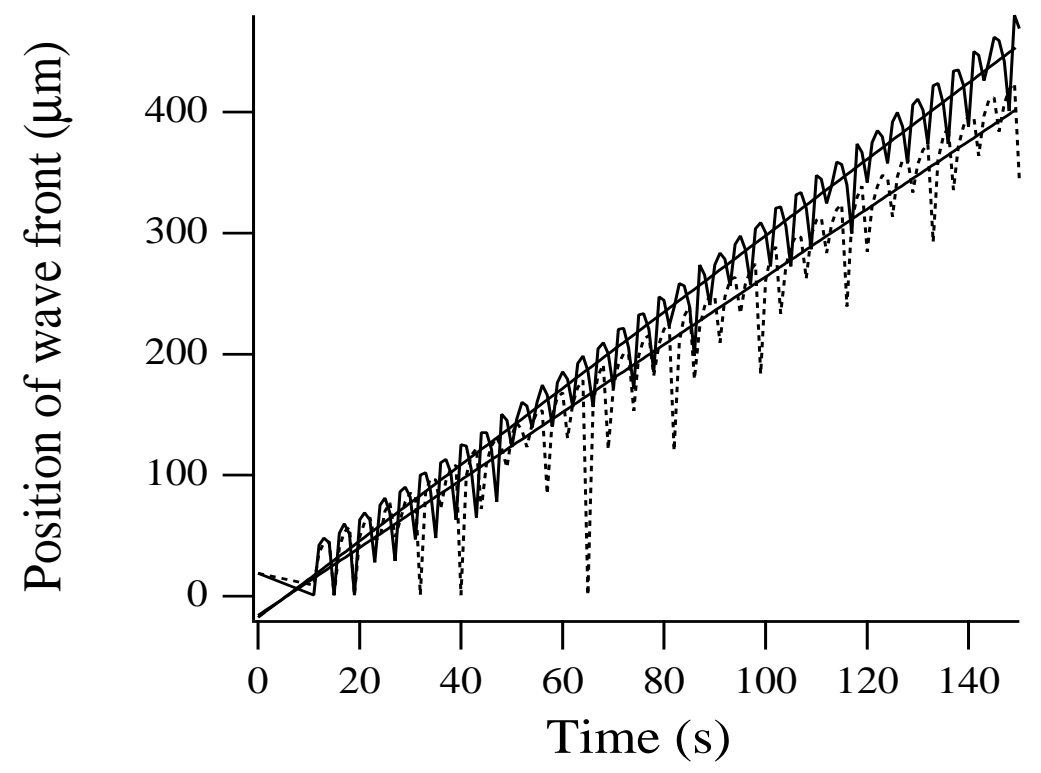

FIG. 10. Position of the wave front as a function of time from the Atri et al. model when $L_{2}=0$. The solid curve corresponds to $D=D_{c}$, and the dotted curve corresponds to $D=0$. The wave front is defined as the place where $c=0.05 \mu \mathrm{M}$, and the wave front speed is defined as the slope of the best-fit line through the points. The computed wave front speeds agree well with the analytical predictions (Fig. 9). 
analysis of Shigesada, Kawasaki, and Teramoto [25] and the form of the solution in the spatially homogeneous case, we look for solutions of the form $u=U(z) \phi(x) e^{i \omega t}$, $v=V(z) \theta(x) e^{i \omega t}$, where $\phi$ and $\theta$ are periodic and $U, V \longrightarrow 0$ as $z \longrightarrow \infty$. The reason for looking for solutions of such a form is not immediately clear. We argue that it is reasonable to expect the wave front to consist of three parts; first, a profile dependent on $z$ that determines the overall wave shape $(U$ and $V$ ); second, a spatially periodic variation caused by the underlying periodicity of the medium ( $\phi$ and $\theta)$; and third, a temporal periodicity caused by the oscillatory nature of the kinetics $\left(e^{i \omega t}\right)$. We emphasize that such a solution will apply only to the wave front, not necessarily to the (nonlinear) periodic waves that occur behind the wave front. Substituting into (30) and (31) gives

$$
\begin{aligned}
& \left(-c \frac{U^{\prime}}{U}+i \omega\right) \phi=\left(\frac{U^{\prime \prime}}{U} \phi+\frac{2 U^{\prime}}{U} \phi^{\prime}+\phi^{\prime \prime}\right)+M f_{u} \phi+M \frac{V}{U} f_{v} \theta, \\
& \left(-c \frac{V^{\prime}}{V}+i \omega\right) \theta=M \frac{U}{V} g_{u} \phi+M g_{v} \theta .
\end{aligned}
$$

It follows that $U^{\prime} / U, U^{\prime \prime} / U$, and $U / V$ must all be constant, and hence

$$
\begin{aligned}
& U=A e^{-s z}, \\
& V=B U
\end{aligned}
$$

for some constants $A, B$, and $s$, where $s$ is complex. Solving for $\phi$ now gives

$$
\phi^{\prime \prime}-2 s \phi^{\prime}+\left(s^{2}-(c s+i \omega)+M f_{u}+\frac{M^{2} f_{v} g_{u}}{(c s+i \omega)-M g_{v}}\right) \phi=0 .
$$

The condition for the existence of a periodic solution to (66) is

(67) $\cosh \left(s\left(L_{1}+L_{2}\right)\right)=\cosh \left(q_{1} L_{1}\right) \cosh \left(q_{2} L_{2}\right)+\frac{q_{1}^{2}+q_{2}^{2}}{2 q_{1} q_{2}} \sinh \left(q_{1} L_{1}\right) \sinh \left(q_{2} L_{2}\right)$,

where

$$
\begin{aligned}
& q_{1}=\sqrt{\frac{(c s+i \omega)^{2}-2(c s+i \omega) \alpha+\alpha^{2}+\omega^{2}}{(c s+i \omega-\alpha)}} \\
& q_{2}=\sqrt{c s+i \omega}
\end{aligned}
$$

This is the same equation given by Shigesada, Kawasaki, and Teramoto but with different definitions for $q_{1}$ and $q_{2}$. We have implicitly assumed that $b_{1}, \ldots, b_{4}=$ 0 and that in the active region (30) and (31) are written in the form of (52) and (53). When $L_{2}=0, c$ can be determined as a function of $\gamma$ from (67), giving the same curve as in Fig. 9 (the $D=0$ curve). We mentioned then that this curve is actually a discontinuous combination of two different branches. As $L_{2}$ is increased, this discontinuity becomes more pronounced, as can be seen from Fig. 11, which shows curves of $c$ versus $\gamma$ for two different values of $L_{2}$. The solid curve corresponds to $L_{1}=50 \mu \mathrm{m}, L_{2}=0.5 \mu \mathrm{m}$, while the dotted curve corresponds to the same value for $L_{1}$ but with $L_{2}=2.5 \mu \mathrm{m}$. This makes it difficult to predict the exact wave front speed merely from consideration of the roots of (67). For instance, when $L_{1}=50$ and $L_{2}=2.5$, the dotted curve in Fig. 11 gives the theoretical prediction of $c$ vs. 


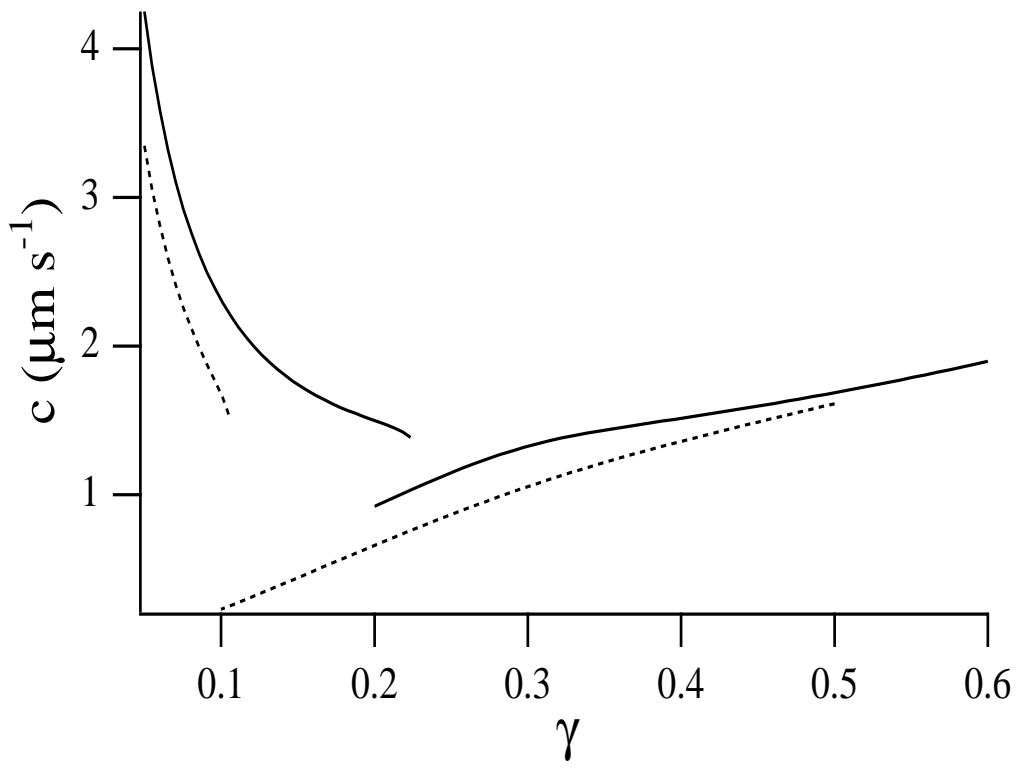

FIG. 11. Wave front speed $c$ as a function of $g$ in the Atri et al. model, calculated from (67). Solid curve: $L_{1}=50 \mu \mathrm{m}, L_{2}=0.5 \mu \mathrm{m}$. Dotted curve: $L_{1}=50 \mu \mathrm{m}, L_{2}=2.5 \mu \mathrm{m}$. Numerical solution of the differential equations gives a wave front speed of $1.49 \mu \mathrm{ms}^{-1}$ in the former case and $1.21 \mu \mathrm{ms}^{-1}$ in the latter. Thus, the theoretical curves do not seem to give a good prediction of the observed wave front speeds.

$\gamma$. However, numerical solution of the differential equations gives a wave front speed of $1.21 \mu \mathrm{m} / \mathrm{s}$, which does not seem to correspond to any particular point on the theoretical curve.

In Figs. 12-14 we show numerical solutions of the Atri et al. model for three cases: $L_{1}=50 \mu \mathrm{m}, L_{2}=0,3$, and $5 \mu \mathrm{m}$. The other parameters were the same in all the runs and were chosen so that the model kinetics had a stable limit cycle. At time $t=0$ a square pulse of $\mathrm{Ca}^{2+}$ was introduced into the left $10 \%$ of the domain, and the solution is plotted between 150 and 200 seconds. The advancing wave front can be clearly seen, and behind the front are periodic plane waves that move away from the wave front at a speed considerably greater than that of the wave front. As it propagates across the domain the wave front does not keep a constant shape, but, as described in Fig. 10, the position of the wave front can still be defined by means of the best-fit line.

It is interesting to note the way in which the solution changes as $L_{2}$ is increased. Clearly, the periodic plane wave behind the wave front is broken into more complex patterns as $L_{2}$ increases, with waves in adjacent active regions moving in opposite directions, and this complexity can result from only relatively small increases in $L_{2}$.

\section{Appendix.}

Equations of the Li-Rinzel model. Details of this model may be found in [13]. The equations are

$$
\frac{\partial c}{\partial t}=D_{c} \frac{\partial^{2} c}{\partial x^{2}}+c_{1}\left(h^{3} v_{1} m_{\infty}^{3}+v_{2}\right)\left(c_{\mathrm{er}}-c\right)-\frac{v_{3} c^{2}}{k_{3}^{2}+c^{2}}
$$




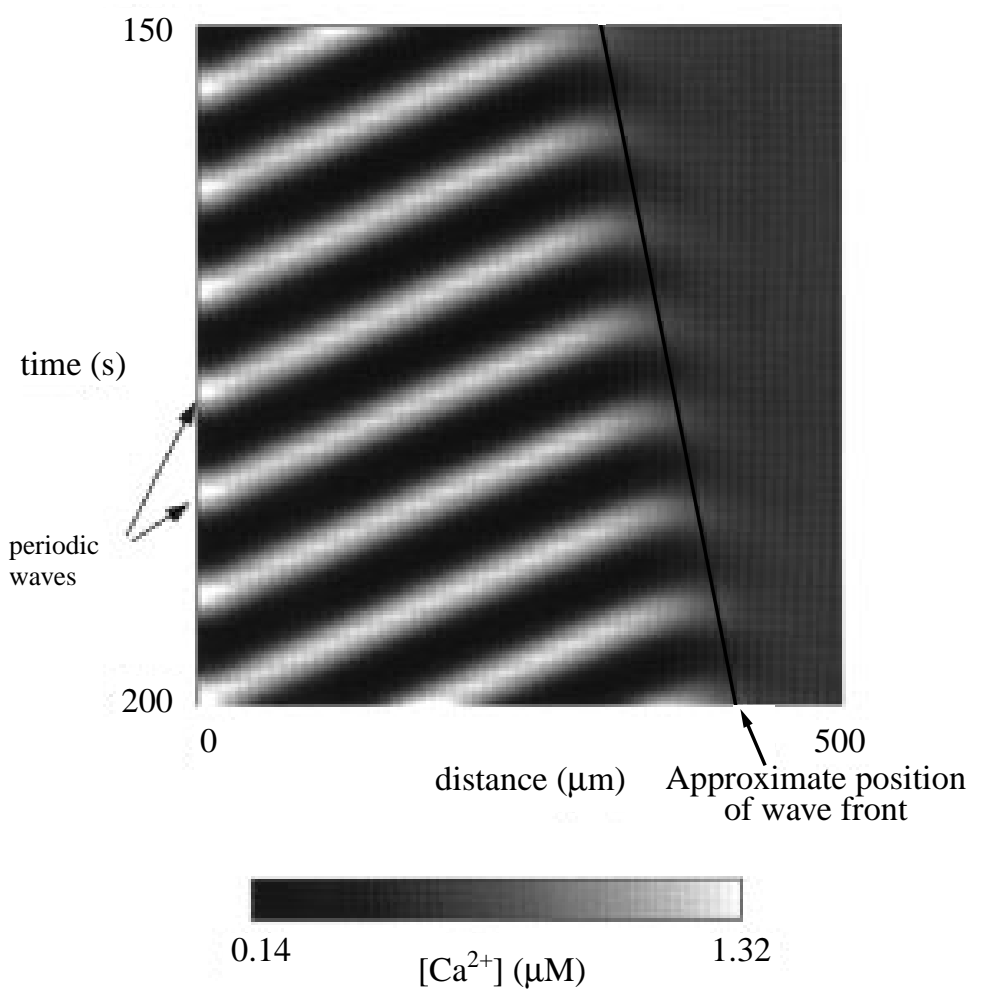

Fig. 12. Plot of solution to the Atri et al. model for $L_{1}=50, L_{2}=0$. The wave front advances slowly from left to right across the domain, and periodic plane waves, moving in the opposite direction with greater speed, appear behind it. The numerical procedure is described in the text. Briefly, at $t=0, u$ was raised on the 10 leftmost spatial grid points. This initiates oscillatory waves in the left hand of the domain, and these oscillatory waves gradually spread over the entire domain, thus creating the wave front.

$$
\begin{aligned}
m_{\infty} & =\left(\frac{\left[\mathrm{IP}_{3}\right]}{\left[\mathrm{IP}_{3}\right]+d_{1}}\right)\left(\frac{c}{c+d_{5}}\right), \\
\tau_{h} \frac{d h}{d t} & =\frac{Q_{2}}{Q_{2}+c}-h \\
Q_{2} & =\frac{d_{2}\left(\left[\mathrm{IP}_{3}\right]+d_{1}\right)}{\left[\mathrm{IP}_{3}\right]+d_{3}}
\end{aligned}
$$

where $c$ denotes $\left[\mathrm{Ca}^{2+}\right.$ ] (for consistency in the text, we denote $\left[\mathrm{Ca}^{2+}\right]$ by $u$ instead of $c), c_{\mathrm{er}}$ denotes the concentration of $\mathrm{Ca}^{2+}$ in the ER and is given by $c_{1} c_{\mathrm{er}}+c=c_{0}$ and where $h$ is the fraction of receptors that have not been inactivated by $\mathrm{Ca}^{2+}$. Here, we used the parameter values from [13], i.e., $c_{0}=2.0 \mu \mathrm{M}, c_{1}=0.185, k_{3}=0.1 \mu \mathrm{M}$, $v_{1}=6 \mathrm{~s}^{-1}, v_{2}=0.11 \mathrm{~s}^{-1}, v_{3}=0.9 \mu \mathrm{Ms}^{-1}, d_{1}=0.13 \mu \mathrm{M}, d_{2}=1.05 \mu \mathrm{M}, d_{3}=$ $0.94 \mu \mathrm{M}, d_{5}=0.082 \mu \mathrm{M}$. All computations were performed with $\left[\mathrm{IP}_{3}\right]=0.3 \mu \mathrm{M}$ and $D_{c}=25 \mu \mathrm{m}^{2} \mathrm{~s}^{-1}$. As discussed in the text, we use only the nullcline $d c / d t=0$, and thus the details of $\tau_{h}$ do not concern us. 

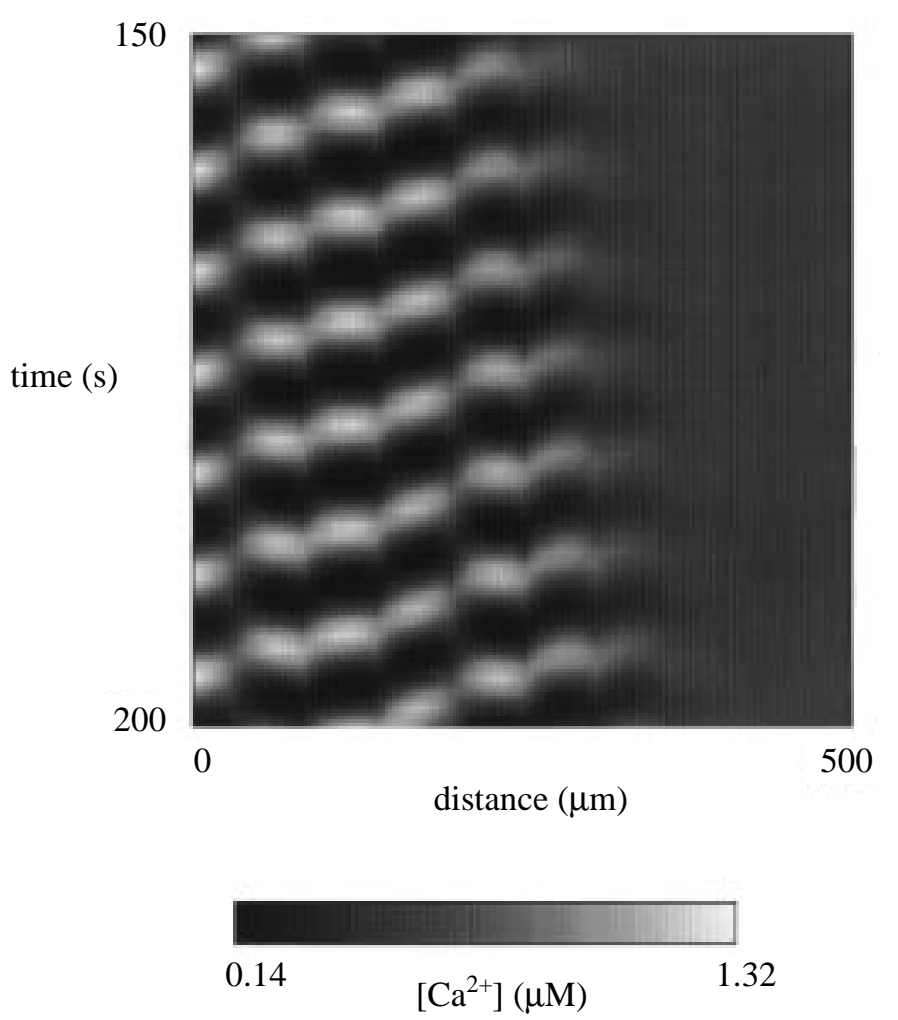

FIG. 13. Plot of solution to the Atri et al. model for $L_{1}=50, L_{2}=3$. The periodic plane waves behind the front are perturbed by the passive regions, but the underlying structure is similar to that seen in Fig. 12.

Equations of the Atri model. Details of this model may be found in [2]. The equations are

$$
\begin{aligned}
\frac{\partial c}{\partial t} & =D_{c} \frac{\partial^{2} c}{\partial x^{2}}+k_{\text {flux }} \mu h\left(b+\frac{(1-b)}{k_{1}+c}\right)-\frac{\gamma c}{k_{\gamma}+c}, \\
\tau_{h} \frac{d h}{d t} & =\frac{k_{2}^{2}}{k_{2}^{2}+c^{2}}-h,
\end{aligned}
$$

where $c$ denotes $\left[\mathrm{Ca}^{2+}\right]$, and $h$ is the fraction of receptors that have not been inactivated by $\mathrm{Ca}^{2+}$. Parameter values used here are $D_{c}=25 \mu \mathrm{m}^{2} \mathrm{~s}^{-1}, b=0.111$, $\gamma=2.2 \mu \mathrm{Ms}^{-1}, \tau_{h}=2 \mathrm{~s}, k_{1}=k_{2}=0.7 \mu \mathrm{M}, k_{\gamma}=0.17 \mu \mathrm{M}, k_{\text {flux }}=8.1 \mu \mathrm{Ms}^{-1}$. In general, $\mu$ is an increasing function of $\left[\mathrm{IP}_{3}\right]$ and is treated as a bifurcation parameter; unless otherwise stated, $\mu=0.7$.

Some computations were performed assuming that $h$ was free to diffuse. In this case the model equations are

$$
\begin{aligned}
\frac{\partial c}{\partial t} & =D_{c} \frac{\partial^{2} c}{\partial x^{2}}+k_{\text {flux }} \mu h\left(b+\frac{(1-b)}{k_{1}+c}\right)-\frac{\gamma c}{k_{\gamma}+c}, \\
\frac{\partial h}{\partial t} & =D \frac{\partial^{2} h}{\partial x^{2}}+\frac{1}{\tau_{h}}\left(\frac{k_{2}^{2}}{k_{2}^{2}+c^{2}}-h\right) .
\end{aligned}
$$




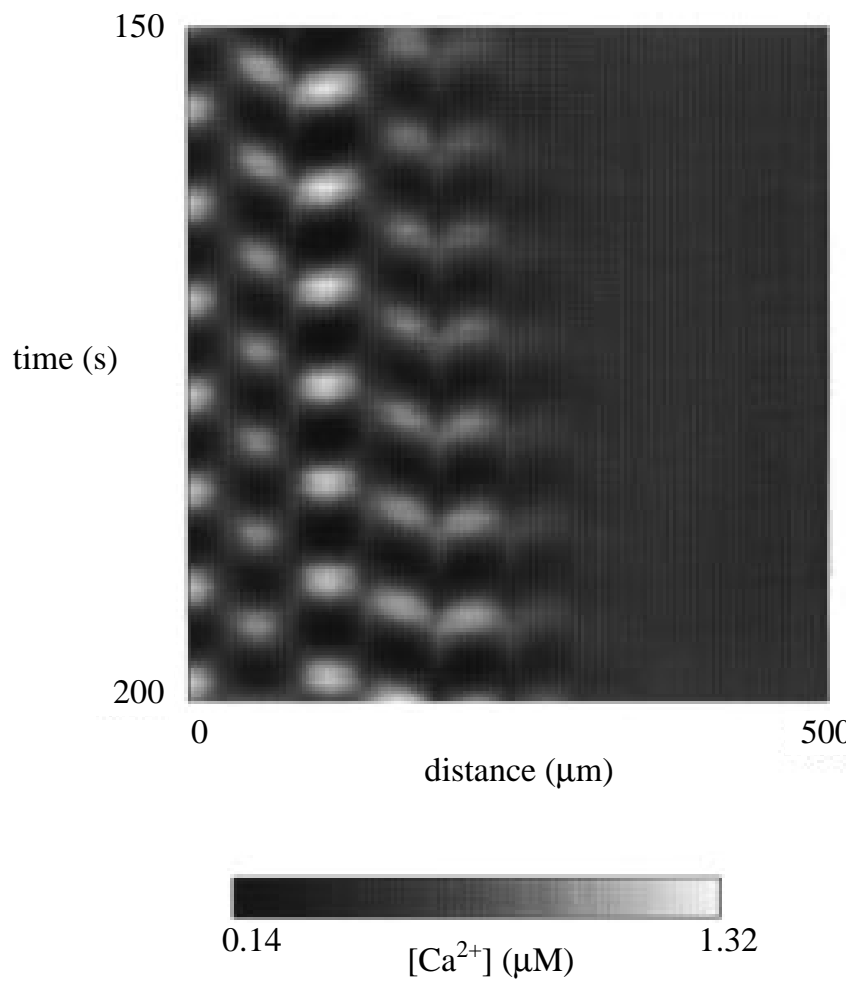

Fig. 14. Plot of solution to the Atri et al. model for $L_{1}=50, L_{2}=5$. The periodic plane waves behind the front are now broken by the passive regions, and adjacent active regions can exhibit periodic plane waves moving in opposite directions. When viewed as a function of time only (i.e., as an animation) this behavior appears disordered.

\section{REFERENCES}

[1] N. L. Allbritton, T. Meyer, And L. Stryer, Range of messenger action of calcium ion and inositol 1,4,5-trisphosphate, Science, 258 (1992), pp. 1812-1815.

[2] A. Atri, J. Amundson, D. Clapham, And J. Sneyd, A single-pool model for intracellular calcium oscillations and waves in the Xenopus laevis oocyte, Biophys. J., 65 (1993), pp. $1727-1739$.

[3] M. J. BERRIDGe, Inositol trisphosphate and calcium signalling, Nature (Lond.), 361 (1993), pp. $315-325$.

[4] G. W. De Young And J. KeIzer, A single pool $\mathrm{IP}_{3}$-receptor based model for agonist stimulated $\mathrm{Ca}^{2+}$ oscillations, Proc. Nat. Acad. Sci. USA, 89 (1992), pp. 9895-9899.

[5] G. Dupont And A. Goldbeter, Properties of intracellular $\mathrm{Ca}^{2+}$ waves generated by a model based on $\mathrm{Ca}^{2+}$-induced $\mathrm{Ca}^{2+}$ release, Biophys. J., 67 (1994), pp. 2191-2204.

[6] R. Fitzhugh, Impulses and physiological states in theoretical models of nerve membrane, in Biological Engineering, H. P. Schwan, ed., McGraw-Hill, New York, 1969.

[7] A. L. Hodgkin And A. F. Huxley, A quantitative description of membrane current and its application to conduction and excitation in nerve, J. Physiol. (Lond.), 117 (1952), pp. $500-544$.

[8] F. C. Hoppensteadt, Mathematical Theories of Populations: Demographics, Genetics and Epidemics, SIAM, Philadelphia, PA, 1975.

[9] A. C. King And D. J. Needham, The effects of variable diffusivity on the development of traveling waves in a class of reaction-diffusion equations, Philos. Trans. Roy. Soc. London Ser. A, 348 (1994), pp. 229-260.

[10] B. Y. Kogan, W. J. Karplus, B. S. Billett, and W. G. Stevenson, Excitation wave propagation within narrow pathways-geometric configurations facilitating unidirectional block and reentry, Phys. D, 59 (1992), pp. 275-296. 
[11] D. A. LARSOn, Transient bounds and time-asymptotic behavior of solutions to non-linear equations of Fisher type, SIAM J. Appl. Math., 34 (1978), pp. 93-103.

[12] M. A. Lewis AND P. GRINDRod, One-way blocks in cardiac tissue: A mechanism for propagation failure, Bull. Math. Biol., 53 (1991), pp. 881-899.

[13] Y.-X. Li AND J. Rinzel, Equations for Ins $P_{3}$ receptor-mediated $\left[\mathrm{Ca}^{2+}\right]$ oscillations derived from a detailed kinetic model: A Hodgkin-Huxley like formalism, J. Theor. Biol., 166 (1994), pp. 461-473.

[14] H. P. MCKeAn, Nagumo's equation, Adv. Math., 4 (1970), pp. 209-223.

[15] S. L. Mironov, Theoretical analysis of $\mathrm{Ca}^{2+}$ wave propagation along the surface of intracellular stores, J. Theor. Biol., 146 (1990), pp. 87-97.

[16] J. D. Murray, Mathematical Biology, Springer-Verlag, Berlin, Heidelberg, New York, 1989.

[17] I. PARKER AND Y. YAO, Regenerative release of calcium from functionally discrete subcellular stores by inositol trisphosphate, Proc. Roy. Soc. London Ser. B, 246 (1991), pp. 269-274.

[18] J. Pauwelussen, One way traffic of pulses in a neuron, J. Math. Biol., 15 (1982), pp. 151-171.

[19] J. Rinzel And J. B. Keller, Traveling wave solutions of a nerve conduction equation, Biophys. J., 13 (1973), pp. 1313-1337.

[20] T. A. Rooney And A. P. Thomas, Intracellular calcium waves generated by Ins $(1,4,5)$ $P_{3}$-dependent mechanisms, Cell Calcium, 14 (1993), pp. 674-690.

[21] M. J. Sanderson, A. C. Charles, S. Boitano, and E. R. Dirksen, Mechanisms and function of intercellular calcium signaling, Mol. Cell. Endocrin., 98 (1994), pp. 173-187.

[22] J. A. Sherratt, The amplitude of periodic plane waves depends on initial conditions in a variety of $\lambda-\omega$ systems, Nonlinearity, 6 (1993), pp. 1055-1066.

[23] J. A. Sherratt, On the evolution of periodic plane waves in reaction-diffusion systems of $\lambda-\omega$ type, SIAM J. Appl. Math., 54 (1994), pp. 1374-1385.

[24] J. A. Sherratt, On the speed of amplitude transition waves in reaction diffusion systems of $\lambda-\omega$ type, IMA J. Appl. Math., 52 (1994), pp. 79-92.

[25] N. Shigesada, K. Kawasaki, And E. Teramoto, Traveling periodic waves in heterogeneous environments, Theor. Pop. Biol., 30 (1986), pp. 143-160.

[26] J. SNEYD, Calcium buffering and diffusion: On the resolution of an outstanding problem, Biophys. J., 67 (1994), pp. 4-5.

[27] J. Sneyd, J. KeIZer, And M. J. SAnderson, Mechanisms of calcium oscillations and waves: A quantitative analysis, FASEB J., 9 (1995), pp. 1463-1472.

[28] J. W. Stucki and R. Somogyi, A dialogue on $\mathrm{Ca}^{2+}$ oscillations: An attempt to understand the essentials of mechanisms leading to hormone-induced intracellular $\mathrm{Ca}^{2+}$ oscillations in various kinds of cells on a theoretical level, Biochem. et Biophys. Acta, 1183 (1994), pp. $453-472$.

[29] J. WAGNER AND J. KeIzer, Effects of rapid buffers on $\mathrm{Ca}^{2+}$ diffusion and $\mathrm{Ca}^{2+}$ oscillations, Biophys. J., 67 (1994), pp. 447-456.

[30] J. X. XIN, Existence and nonexistence of traveling waves and reaction-diffusion front propagation in periodic media, J. Stat. Phys., 73 (1993), pp. 893-926. 